Archives de sciences sociales des religions

176 | octobre-décembre 2016

Bulletin Bibliographique

\title{
Le ciel sur la terre ? Cinquante ans de liturgie catholique
}

Alain Rauwel

\section{OpenEdition}

1 Journals

Édition électronique

URL : http://journals.openedition.org/assr/28148

DOI : $10.4000 /$ assr.28148

ISSN : 1777-5825

Éditeur

Éditions de l'EHESS

\section{Édition imprimée}

Date de publication : 31 décembre 2016

Pagination : 231-240

ISSN : 0335-5985

\section{Référence électronique}

Alain Rauwel, «Le ciel sur la terre ? Cinquante ans de liturgie catholique », Archives de sciences sociales des religions [En ligne], 176 | octobre-décembre 2016, mis en ligne le 01 janvier 2019, consulté le 04 janvier 2020. URL : http://journals.openedition.org/assr/28148; DOI : 10.4000/assr.28148 


\title{
Alain Rauwel \\ Le ciel sur la terre ? \\ Cinquante ans de liturgie catholique
}

\author{
À propos de : \\ DE BREMOND D'ARS Nicolas, La liturgie catholique: quarante ans de \\ pratiques en France, préface de D. Hervieu-Léger, Rennes, Presses \\ Universitaires de Rennes, coll. "Sciences des religions ", 2015, \\ $216 \mathrm{p}$. \\ BugnINI Annibale, La réforme de la liturgie (1948-1975), trad. P.-D. Nau \\ et Ph. de Lacvivier, Paris-Perpignan, Desclée de Brouwer, 2015, \\ $1034 \mathrm{p}$. \\ Cassingena-Trévedy François, Les Pères de l'Église et la liturgie, Perpi- \\ gnan, Artège, 2016 ( $2^{\mathrm{e}}$ éd.), $388 \mathrm{p}$. \\ Chiron Yves, Annibale Bugnini, Paris, Desclée de Brouwer, 2016, \\ $221 \mathrm{p}$.
}

En une grosse décennie, entre 1960 et 1970, l'Église latine a connu une révolution culturelle de première grandeur, certes préparée de longue date, certes multiforme, mais dont la dimension la plus visible (au sens fort que prend cet adjectif quand l'ecclésiologie est en jeu) reste le virage à 180 degrés de sa liturgie, passée en quelques années d'une mystérialité sacrale largement ésotérique à une logique de rassemblement vernaculaire. Il n'est pas certain que, malgré les avertissements des plus lucides ${ }^{1}$, sociologues et historiens des religions aient consacré à l'observation du phénomène in vivo toute la diligence requise. Des entretiens pourraient encore pallier cette lacune, mais seulement en partie, car l'iter du catholicisme a été tel en un demi-siècle qu'il est devenu impossible de ne pas relire 1969 à la lumière de 2016. Restent au moins les données institutionnelles, que le grand âge des acteurs, l'approche de la mort, l'angoisse de la trahison ont contribué à divulguer largement ces derniers temps.

Parmi ces acteurs, c'est-à-dire ceux qui ont fait la réforme liturgique catholique, dans les officines vaticanes, les instituts spécialisés, les cénacles d'experts,

1. J. Séguy, "Suggestions pour une sociologie des liturgies chrétiennes ", Archives de sciences sociales des religions, $\mathrm{n}^{\circ} 22,1966, \mathrm{p} .145-151$.

ARCHIVES DE SCIENCES SOCIALES DES RELIGIONS 176 (octobre-décembre 2016), p. 231-239 
nul n'a eu plus de poids qu'Annibale Bugnini. De 1948 à sa disgrâce en 1975, de Pacelli à Montini, Bugnini fut au cœur du vortex rituel. Avant, pendant et après Vatican II, tout ce qui a été promulgué en matière de culte par l'autorité romaine est passé par ses mains et porte sa griffe. Il est probable que si, comme cela semblait écrit, Bugnini était mort cardinal dans un bel appartement de la via della Conciliazione, il aurait emporté dans son tombeau de marbre ses souvenirs et ses secrets. La médiocrité du personnage était bien faite pour assurer son succès en Curie : fils de paysans repéré pour sa piété, prêtre lazariste, tout dévoué à l'institution ecclésiastique et à ses princes, il n'a pour ainsi dire pas d'histoire personnelle, comme le montre la "biographie " que lui consacre Yves Chiron. Rédigée avec compétence, rigueur et probité, elle est, bien plutôt qu'une enquête sur les motivations d'un homme qui apparaît comme presque transparent, un résumé de son œuvre - résumé d'ailleurs précieux en ce qu'il permet de prendre connaissance en 200 pages aérées du contenu d'une documentation bavarde et fastidieuse.

Felix culpa pour l'historien, il est arrivé à Bugnini ce qui arrive souvent dans les sociétés de cour à l'agonie : du jour au lendemain, sans explication, il s'est trouvé démis de toutes ses charges et exilé usque ad mortem, sous des apparences de promotion, à l'autre bout du monde - en l'espèce la nonciature de Téhéran. On devrait bien consacrer une étude aux modalités de création et de transmission de la légende urbaine qui, se propageant comme une traînée de poudre dans la catholicité aux abois des dernières années de Montini, a justifié la disgrâce de Bugnini par la révélation de son appartenance supposée à la franc-maçonnerie : Les caves $d u$ Vatican semblent ici en dessous de la réalité ! Le bouillant P. Bouyer, dont on a attendu dix ans la (mauvaise) publication des Mémoires, a tenu sa partie en qualifiant le secrétaire du Consilium de "scélérat douteux [...] aussi dépourvu de culture que de simple honnêteté ${ }^{2}$ ». Toujours est-il que l'obsession du nonce malgré lui est devenue de faire connaître urbi et orbi son intégrité et ses mérites, d'où la rédaction de mémoires publiées à titre posthume, et surtout d'une somme de plus de mille pages, La réforme liturgique (1948-1975), parue en italien en 1983 et dont on espérait depuis lors la traduction française, que viennent de donner les éditions Desclée de Brouwer.

La réforme liturgique est un ouvrage de pure apologétique, dont la fin est entièrement d'autojustification; nonobstant, il est indispensable sur les étagères de quiconque s'intéresse à la liturgie contemporaine, en raison de la masse considérable de documents que Bugnini brasse et souvent cite en tout ou partie. La

2. L. Bouyer, Mémoires, Paris, Éditions du Cerf, coll. "Bibliothèque du Cerf », 2014, p. 198. Bouyer fait de Bugnini un napolitain, alors qu'il était ombrien. L'annotateur de l'édition ne rectifie pas. Des « compléments » oubliés aux mémoires viennent de paraître, deux ans après, chez un autre éditeur (L. Bouyer, Lectures et voyages. Compléments aux Mémoires, Paris, Ad Solem, 2016)! 
démarche est analytique d'un bout à l'autre. L'auteur examine la procédure générale de la réforme, les principes communs, les réalisations en matière de messe, d'office, de sacrements, de bénédictions, de musique... Pour chaque thème, il narre selon la chronologie la suite des réunions, des courriers échangés, des versions successives. Peu de choses, sans doute, étaient entièrement inédites dans cette masse. Mais il fallait pour les retrouver consulter des comptes rendus officiels difficiles à trouver en dehors de Rome, des récits dispersés de témoins, des gloses signées de savants qui avaient prêté la main aux rédactions post-conciliaires et qui, au détour d'une note, avouaient à demi-mot les tenants et aboutissants de tel ou tel choix. Dans la Summa bugniniana, tout est commodément rassemblé.

Il en ressort que la révision générale à laquelle ont été soumis les livres de la liturgie latine après 1962 a été pour l'essentiel une affaire d'experts (periti), c'est-à-dire de professeurs. Il n'allait pas de soi que les évêques, que Vatican II définissait au même moment comme investis de la "plénitude du sacerdoce ", abandonnent la manifestation principale de leur autorité à des hommes de cabinet. Mais le $\mathrm{Xx}^{\mathrm{e}}$ siècle a vu rejouer la même pièce qu'après le concile de Trente, à la fin duquel les Pères avaient remis au pape la responsabilité de l'édition des livres cultuels - au pape, c'est-à-dire à un petit cercle de lettrés et de prélats de Curie. L'érudition textuelle a été investie dans les deux cas du statut de critère ultime. La différence est double, cependant. D'abord, il ne s'agissait pas après Vatican II du simple toilettage centralisateur promulgué par Pie V, mais bien d'une reprise en sous-œuvre de tout le système, entraînant des modifications substantielles de la plupart des actions rituelles. Ensuite, si les chevilles ouvrières ne pouvaient être, encore à ce moment, qu'italiennes, à l'exemple d'un Bugnini, rompu à tous les jeux de couloirs des palais d'outre-Tibre, La réforme liturgique montre à quel point les têtes pensantes ont été en très grande partie françaises, ou plutôt francophones. La besogne du Consilium ad exsequendam constitutionem de sacra liturgia a été en effet méthodiquement répartie entre toute une série de groupes de travail, dont la composition complète est indiquée à chaque fois. Les ténors du "Mouvement liturgique » franco-belge y tiennent la part du lion, derrière les Martimort, Gy, Botte... qui fourbissaient leurs armes depuis longtemps.

Tant s'en faut, pour autant, qu'un boulevard se soit ouvert devant leurs initiatives. Bugnini laisse entendre quelle fut la part des rivalités internes à la Curie dans la gestion d'une question sensible entre toutes et très médiatisée. La vieille Congrégation des rites ne décolérait pas d'avoir été dépossédée de l'initiative, et ne se privait pas de contrer le Consilium ${ }^{3}$. L'ex Saint-Office, devenu Congrégation pour la doctrine de la foi et resté sous la présidence de l'irascible Ottaviani jusqu'en 1968, entendait se prononcer à chaque fois que la lex credendi

3. On possède à ce sujet les notes du cardinal Antonelli : N. Giampietro, Le cardinal Antonelli et les développements de la réforme liturgique de 1948 à 1970, Versailles, Artège, coll. "Liturgie ", 2004. 
était en cause - c'est-à-dire tout le temps. La Secrétairerie d'État cherchait à garder le leadership sur toute l'activité de la machine ecclésiastique. De plus menus organes ne se privaient pas de faire aussi beaucoup de bruit, comme les chapelles musicales, voyant dans la floraison de cantiques exigée par la participatio actuosa une menace pour les techniques de spécialistes dont elles étaient les conservatrices. D'où jeux de diplomatie cléricale (comme lorsqu'il fallut tenir compte dans la révision du bréviaire de la version latine du psautier signée par le cardinal Béa et unanimement considérée comme un échec), lettres sans réponses, commissions mixtes, et quelques enterrements de première classe.

Pour trancher, Bugnini a joué en continu la carte de l'Autorité, avec majuscule : celle du pape, c'est-à-dire de Montini pendant toute la phase de réforme. Sur le fonctionnement réel de la primauté dans le système latin, le cas liturgique offre un excellent terrain d'observation. Montini veut tout savoir des travaux préparatoires. Il lit, la plume à la main, rapports et propositions. Sa pente le porte à soutenir l'action modernisatrice du Consilium et des experts. Mais chez ce perpétuel torturé ${ }^{4}$, point de pente qui n'ait sa contre-pente. Il s'émeut de suppressions ou de modifications, demande qu'on ne touche pas à telle ou telle formule. Selon le conseiller qui a parlé en dernier, il s'incline devant l'autorité des savants ou transforme la demande en ordre, rarement exprimé directement. Le statut de clef de voûte du pape apparaît bien au fil de ces crises quasi quotidiennes : il est celui qui maintient l'édifice, mais cela n'est possible qu'en recevant les poussées contradictoires de tous les segments. Aussi finit-on par ne plus bien savoir qui décide, tant les influences croisées s'accumulent dans le processus de décision. Autorité personnelle et fonctionnelle, charisme, technostructure, prophétisme et fonctionnariat : tout cela est en jeu dans l'affaire de la réforme.

Dans l'opinion, catholique ou non, le test de la réforme a été ce que l'on a alors appelé « la nouvelle messe" (c'est le titre d'un chapitre de Chiron). La réforme liturgique et la biographie de son auteur racontent dans le détail comment a été élaboré le nouvel Ordo entré en vigueur au premier dimanche de l'Avent 1969. On y lit notamment le récit des "messes normatives ", c'est-à-dire des célébrations expérimentales à huis clos destinées à recueillir l'avis des spécialistes et des prélats sur la mise en œuvre des nouvelles propositions : étranges objets que ces performances rituelles dont les participants n'étaient pas convoqués pour un acte religieux, mais pour une évaluation critique. Les impressions furent d'ailleurs loin d'être unanimement positives. S'exprimait largement une inquiétude face à une manière de toucher l'intouchable qui, pour certains, confinait au sacrilège. Dès ce tout début de la réforme, la valse-hésitation de l'institution romaine, obligée de poursuivre dans une voie qu'elle avait elle-même ouverte tout en entendant aller le moins loin possible, voire n'aller nulle part, est un spectacle

4. Dont Yves Chiron a écrit aussi la biographie : Paul VI, le pape écartelé, Paris, Perrin, 1993. 
fascinant. Les nombreuses concessions qui s'imposèrent pour aboutir expliquent sans doute pourquoi, sur le moment comme à distance, le constat d'échec s'impose à beaucoup d'observateurs.

\section{$* *$}

On l'a très justement fait observer : le retour ad pristinam Ecclesiae formam dont la génération de Vatican II a fait son leitmotiv n'était pas, en dépit des effets d'affichage, un retour à l'âge apostolique proprement dit - trop ouvert encore, trop informel -, mais bien un retour à l'Église déjà institutionnalisée, cléricalisée et ritualisée du $\mathrm{III}^{\mathrm{e}}-\mathrm{IV}^{\mathrm{e}}$ siècle. Dans un domaine comme la liturgie, le « retour aux Pères " fut donc nettement plus décisif que le retour aux Écritures. Cela rend d'autant plus précieux un compendium de la pensée patristique sur le culte, comme celui qu'a donné en 2009 François Cassingena-Trévedy, aujourd'hui réédité avec une chaleureuse préface de Michel-Yves Perrin. L'objectif de l'auteur est de saisir "l'acte de célébrer » au fil d'un ample dépouillement des écrits patristiques, et de tracer ainsi un «profil comportemental du sujet liturgique » ancien. C'est bien en effet une anthropologie d'homo liturgicus qui est visée, ce qui n'est pas tout à fait une phénoménologie du culte à l'âge post-apostolique. Le relatif inintérêt pour les lieux de rassemblement en est une preuve, et en même temps une limite: dans un geste hautement significatif comme celui de la prière orientée, le conversi ad Dominum vaut pour les «pierres vivantes » comme pour les pierres à bâtir, et la pointe de l'analyse est au croisement des deux dynamiques ${ }^{5}$.

L'auteur met bien en lumière la polarité, devenue avec le temps une tension, entre la volonté des liturges de la terre de s'unir à ceux du ciel, singulièrement aux anges, en une acclamation non seulement partagée, mais tendanciellement fusionnelle ${ }^{6}$, et la nécessité de promouvoir la cohérence éthique de l'engagement religieux en faisant de la philanthropia l'issue normale de l'office. Le postulat de ce travail scientifique qui se veut aussi d'édification est que les siècles patristiques offrent un modèle d'équilibre entre présence au monde et anticipation des eschata.

Les notations et citations très nombreuses donnent tout son prix à un ouvrage qui se désigne lui-même comme "encyclopédie liturgique "; les catégories retenues sont-elles pour autant toujours bien pertinentes ? Cassingena tient à la définition du culte comme " fête spirituelle ". À y bien regarder, ce sont pourtant d'autres champs sémantiques qui sont ouverts par le vocabulaire ancien. Sollemnitas induit un mode d'être, d'agir et de dire qui a très peu en commun avec ce

5. Comme l'a parfaitement réalisé M. Metzger dans son ambitieuse et riche synthèse, L'Église dans l'Empire romain - Le culte - Volume I : Les institutions, Rome, Pontificio Ateneo Sant'Anselmo, coll. "Studia anselmiana », 163 - « Analecta liturgica », 33, 2015.

6. Il est curieux que Cassingena ne fasse pas figurer dans sa bibliographie les travaux classiques de Peterson, qui apportent beaucoup d'eau à son moulin. 
qui vient à l'esprit d'un lecteur moderne rencontrant le mot «fête » - sans compter que personne aujourd'hui ne dissociera une réflexion sur la fête des apports de l'anthropologie et de la sociologie, particulièrement nombreux sur ce point ${ }^{7}$ : Cassingena ne s'y refuse pas, mais s'en tient à des généralités fort peu heuristiques (Wunenburger), voire désagréablement connotées (Eliade). C'est une lacune incontestable de son travail. Il faut sans doute en chercher la source dans un rapport assez flottant à l'histoire. Le souhait d'inscrire les formes cultuelles de l'Antiquité tardive dans les milieux sociaux où elles se sont développées est affiché, et dans une certaine mesure mis en œuvre. Dans la lignée des recherches de Philippe Bernard sur la "liturgie de la Victoire ${ }^{8}$ ", le lien entre le cérémonial des basiliques et celui de la cour impériale est bien mis en valeur. Mais les historiens mobilisés sont largement $a d$ hoc : à un Peter Brown et à son humour anglo-saxon si efficacement désacralisateur, Cassingena préfère les échos d'harmonium et l'onction toute prélatice d'un Marrou. Rien d'étonnant à ce qu'il affirme rechercher la "paideia liturgique » des Pères : c'est marcher dans les traces du maître.

Reste pour nous une conclusion, qui était déjà en 2007 celle du livret Te igitur ${ }^{9}$, et que les dernières pages présentent avec une grande clarté. Cassingena y distingue deux « ethos liturgiques ", rattachés symboliquement l'un à Chrysostome, l'autre à Augustin. Le premier est plus sensible à la majesté divine, à l'extratemporalité du culte, à la hiérarchie des acteurs; il est heureusement qualifié de " panliturgisme ». L'autre est plus intéressé à mettre le rite en cohérence avec la vie, à reconnaître dans la foi l'humble passage du Christ pascal. Même le plus distant des observateurs de l'Église contemporaine identifiera l'éthos augustinien à une conception « conciliaire » de la liturgie (ce que confirme Cassingena) et le mode chrysostomien à une conception " ratzingerienne " (ce que l'auteur n'avoue pas). Il n'y a pas nécessairement opposition frontale entre ces deux modèles, mais il y a incontestablement deux styles, deux manières d'habiter la maisonÉglise ${ }^{10}$. Laquelle s'imposera, si tant est qu'il doive y avoir un vainqueur et un vaincu? L'enjeu n'est pas mince.

7. F.-A. Isambert, Le sens du sacré: fête et religion populaire, Paris, Éditions de Minuit, 1982.

8. "La liturgie de la victoire : mise en scène du pouvoir, Ordo Missae et psalmodie responsoriale dans l'Antiquité tardive et le haut Moyen Âge. Réflexions à partir de l'Expositio du Pseudo-Germain de Paris ", Ecclesia Orans, 13, 1996, p. 349-406.

9. F. Cassingena-Trévedy, Te igitur : le missel de s. Pie V, Gèneve, Ad Solem, 2007.

10. La réflexion sur la religion comme style, esquissée par Balthasar sous un angle particulier, doit sa systématisation à C. Theobald, Le christianisme comme style, 2 vol., Paris, Éditions du Cerf, coll. "Cogitatio fidei », 260-261, 2007. En ecclésiologie, l'historien J. O’Malley a proposé aussi une analyse des paradigmes tridentin et moderne en termes de style: "Trent and Vatican II: two styles of Church ", From Trent to Vatican II: historical and theological investigations, New York, Oxford University Press, 2006, p. 301-320. 
Qu'en est-il donc, "cinquante ans après " ? Le papier noirci sur la question ne manque pas, mais ne mérite la plupart du temps guère d'intérêt. Entre les lamentations des Jérémie ratzingeriens ou lefebvristes sur les ruines du Temple et les enthousiasmes de commande des fonctionnaires ecclésiastiques sur la régénération du culte, c'est l'analyse qui fait défaut. Il faut d'autant plus saluer le courage de Nicolas de Bremond d'Ars qui, sous un titre très vaste, n'a entrepris rien moins que d'offrir une sociologie générale de la liturgie telle qu'elle est pratiquée aujourd'hui dans les communautés locales de l'Église catholique romaine hâtons-nous de préciser : en France, et même dans le milieu urbain français, ce qui a son importance. Non pas dans une paroisse particulière, non pas dans un groupe bien délimité, non pas pour un certain type de célébrations, mais à l'échelle d'une post-chrétienté entière. L'auteur est placé pour cela à un carrefour de compétences à la fois favorable et périlleux : sociologue des religions, ayant derrière lui une œuvre déjà importante, et en même temps prêtre catholique, donc acteur privilégié de ce qu'il analyse. Il faut lui savoir gré de ne pas s'être laissé paralyser par des scrupules méthodologiques excessifs, et d'avoir osé proposer un panorama qui était très attendu, comme le souligne Danièle HervieuLéger dans sa préface. Beaucoup d'études microscopiques d'événements rituels ponctuels exigeaient en effet d'être replacées dans un cadre global d'intelligibilité, tout comme beaucoup de réflexions plus ou moins informées sur le destin récent de la liturgie pêchaient par l'absence d'une définition précise de ce dont il est question. Une telle définition appelle, en bonne méthode, une présentation initiale des lieux, des gens, des mots, des objets... C'est l'une des forces du livre que de n'avoir pas reculé devant l'extrême difficulté d'une bonne description des actions rituelles. À mi-chemin entre une extériorité applatissante et une connivence excluante, il trouve souvent l'équilibre subtil qui permet de donner à voir une vigile pascale, une cérémonie d'obsèques, un baptême, une messe dominicale, une ordination...

Tous les rites décrits s'accomplissent dans un cadre spatio-temporel, dont l'analyse est au cœur de la proposition de N. de Bremond d'Ars. Tout comme les historiens de l'Église ont suivi, ces dernières années, le fil rouge de la spatialité, le sociologue, à chaque étape de son " parcours liturgique ", s'intéresse aux rapports entre nef et sanctuaire, c'est-à-dire entre deux groupes numériquement inégaux d'acteurs dont le placement soigneusement différencié exprime la différence fonctionnelle, voire ontologique, et peut-être politique. Au point de convergence des regards et des mouvements, il note la stature axiale de l'autel, dont les difficultés d'usage et de perception mettent selon lui en lumière les apories d'un système largement inabouti ${ }^{11}$. On relèvera aussi ses excellentes remarques sur l'église comme espace sonore, c'est-à-dire aujourd'hui sonorisé - une pratique

11. Sur les questions de "mise en espace ", on pourra relire les réflexions très fines proposées, à la fois " à chaud » et à distance, par le talentueux ritologue qu'était Jean-Yves Hameline, rééditées dans Petite poétique des arts sacrés, Paris, Éditions du Cerf, coll. « Lex orandi », 2014. 
qui va bien au-delà de la simple commodité ${ }^{12}$. Avec originalité, l'auteur aborde encore la question de la temporalité. Partant du constat selon lequel la late modernity se caractérise par l'accélération du temps de la vie individuelle, à l'échelle duquel sont désormais incluses des mutations qui occupaient aux siècles précédents plusieurs générations, il diagnostique une incompatibilité de ce rapport au temps avec celui de l'institution, distinguée par la lenteur d'une structure millénaire qui se présente comme ayant un pied dans l'éternité. En ce hiatus réside l'incapacité désormais acquise du rituel catholique à structurer encore le temps social. Et l'observateur attentif de la liturgie de voir là une clef d'intelligibilité majeure pour la "crise de la pratique " mesurée jusqu'à plus soif par enquêtes et sondages : s'il s'agissait, bien plus que de croyances acceptées ou refusées, de rythmes et d'incompatibilités rythmiques?

Sans langue de bois ni langue de buis, N. de Bremond d'Ars constate donc des échecs du processus de ritualisation, comme celui de ce que l'on a longtemps appelé " confession ", qui est devenu " pénitence » (ou " réconciliation ») et qui peine à prendre vraiment forme, rejoignant selon l'auteur le sacrement des malades dans un "échec liturgique des thérapies». Les précisions de Bugnini sont ici utiles ${ }^{13}$ : il indique combien la réforme de la pénitence fut une des plus laborieuses, sans cesse ramenée par les gardiens des formulations néo-scolastiques à une casuistique judiciaire peu compatible avec la volonté de prôner une réinsertion globale dans l'Ecclesia par le repentir et le ferme propos ${ }^{14}$. Ce faisant, il laisse entendre que les livres publiés sont en retrait de ce qui aurait d'abord été souhaité. L'évaluation suggérée par le curialiste et revendiquée par le sociologue va dans le sens d'une réforme impossible, par définition toujours insuffisante pour ceux qui espèrent le plein ralliement du catholicisme aux valeurs de la modernité et toujours déjà abusive pour les tenants du semper idem.

N. de Bremond d'Ars franchit un seuil en introduisant dans une deuxième partie de son livre ( «La machine de gloire») un concept destiné à rassembler les nombreuses et riches notations du parcours. Ce concept, très influencé par Giorgio Agamben ${ }^{15}$, est précisément celui de "gloire » au sens très spécifique de l'hébreu biblique kabod et du grec patristique doxa: retour, sous une forme très différente, au lexique des Pères. On dira que l'idée est ancienne, si l'on se réfère à la finalité proprement eschatologique du culte comme anticipation du Royaume. Mais ce n'est pas uniquement cela que vise l'auteur. Pour lui, il y a

12. Évaluation sans concession des pratiques de sonorisation dans la thèse d'O. Manaud, La musique liturgique édifie l'Église, Paris, Pierre Tequi, coll. "Croire et savoir », 2013, p. 231-241.

13. La réforme de la liturgie, p. 708-728.

14. L'un des tenants de la signification collective de la réconciliation sacramentelle dans les années 1960 était le jeune théologien Josef Ratzinger !

15. Le Règne et la gloire, Paris, Éditions du Seuil, coll. "L'ordre philosophique », 2008 ( « Homo sacer », II, 2). 
déjà production de gloire dans l'bic et nunc de la célébration, qui exprime à la fois la gloire du destinataire transcendant et celle des fidèles rassemblés, considérés aussi bien individuellement qu'en corps : gloire déjà là, sacramentellement acquise, et gloire promise, selon la dialectique de l'advenu et de l'à-venir qui structure l'ensemble du système chrétien. N. de Bremond d'Ars, qui avait commencé son travail par un acte d'audace, le termine de même : en important dans les sciences sociales un concept forgé par des siècles d'élaborations théologiques. Certains pourront préférer entrer dans le vaste champ des études rituelles par d'autres portes, mais qui contestera qu'il est bienfaisant, par un effet de translatio studiorum, de susciter un regard neuf et fécond sur des réalités trop souvent négligées ou mal observées ?

Alain RAUWEL

Centre d'études en sciences sociales du religieux (CéSor, EHESS-CNRS) a.rauwel@wanadoo.fr 
